\title{
Nouveaux arrivants de 1989, langue et mobilité professionnelle sur le marché du travail de Montréal : une approche longitudinale
}

\section{Language and occupational mobility in the Montréal labour market for immigrants arriving in 1989: A longitudinal approach}

\section{Ronald Lebeau et Jean Renaud}

Volume 31, numéro 1, 2002

L’immigration

URI : https://id.erudit.org/iderudit/000424ar

DOI : https://doi.org/10.7202/000424ar

Aller au sommaire du numéro

Éditeur(s)

Association des démographes du Québec

ISSN

0380-1721 (imprimé)

1705-1495 (numérique)

Découvrir la revue

Citer cet article

Lebeau, R. \& Renaud, J. (2002). Nouveaux arrivants de 1989, langue et mobilité professionnelle sur le marché du travail de Montréal : une approche longitudinale. Cahiers québécois de démographie, 31(1), 69-94.

https://doi.org/10.7202/000424ar

\section{Résumé de l'article}

L'établissement économique des nouveaux arrivants est souvent perçu comme un effet de leur capacité de parler la langue du pays d'accueil. Cette hypothèse, simple et intuitive, doit maintenant être démontrée : la plupart des études n'établissent pas de relation entre la langue et la situation sur le marché de l'emploi. De plus, la recension des écrits ne nous fournit pas toujours des preuves empiriques et solides de l'effet des habiletés linguistiques. Cet article vise à réexaminer cette question. L'examen est centré sur les trois premières années d'établissement seulement, et repose sur un échantillon pris dans une région bilingue afin de distinguer l'effet de la maîtrise des langues de l'effet social de la langue. La langue a-t-elle des effets sur la mobilité professionnelle (temps mis pour accéder à un emploi et durée de séjour en emploi) ? Elle a un effet sur la mobilité, mais qui se modifie selon le rang de l'emploi : elle écourte le séjour dans le premier emploi et allonge la durée du troisième. La nature de l'effet se modifie également avec le temps : si les compétences linguistiques en soi semblent jouer un rôle au début, cet effet disparaît plus tard pour faire place à l'effet social de la langue.
Tous droits de reproduction, de traduction et d'adaptation réservés (C) Association des Démographes du Québec, 2002
Ce document est protégé par la loi sur le droit d'auteur. L'utilisation des services d'Érudit (y compris la reproduction) est assujettie à sa politique d'utilisation que vous pouvez consulter en ligne. 
Cahiers québécois de démographie

Vol. 31, no 1, printemps 2002, p. 69-94.

\title{
Nouveaux arrivants de 1989, langue et mobilité professionnelle sur le marché du travail de Montréal : une approche longitudinale
}

\author{
Ronald LEBEAU et Jean RENAUD *
}

L'établissement économique des nouveaux arrivants est souvent perçu comme un effet de leur capacité de parler la langue du pays d'accueil. Cette hypothèse, simple et intuitive, doit maintenant être démontrée : la plupart des études n'établissent pas de relation entre la langue et la situation sur le marché de l'emploi. De plus, la recension des écrits ne nous fournit pas toujours des preuves empiriques et solides de l'effet des habiletés linguistiques. Cet article vise à réexaminer cette question. L'examen est centré sur les trois premières années d'établissement seulement, et repose sur un échantillon pris dans une région bilingue afin de distinguer l'effet de la maîtrise des langues de l'effet social de la langue. La langue a-t-elle des effets sur la mobilité professionnelle (temps mis pour accéder à un emploi et durée de séjour en emploi) ? Elle a un effet sur la mobilité, mais qui se modifie selon le rang de l'emploi: elle écourte le séjour dans le premier emploi et allonge la durée du troisième. La nature de l'effet se modifie également avec le temps : si les compétences linguistiques en soi semblent jouer un rôle au début, cet effet disparaît plus tard pour faire place à l'effet social de la langue.

English abstract, p. 93.

$\mathrm{L}^{\mathrm{a}}$ a langue est souvent considérée comme un facteur clé du processus d'intégration des immigrants. Plusieurs études tendent en effet à démontrer que la connaissance de la langue du pays d'accueil a un effet important sur la capacité d'entrer dans le marché du travail de ce pays et d'y rester (McAllister, 1986; Majka et Mullan, 1991; Kossoudji et Cobb-Clark, 1996; Drinkwater et O'Leary, 1997; Shu, 1996; Bloom et Grenier, 1996; Stolzenberg et Tienda, 1997; Amissah, 1996; Miller et Neo, 1997). Toutefois, d'autres études tendent à démontrer le

* Département de sociologie et Centre d'études ethniques, Université de Montréal. Cet article est tiré d'un mémoire de maîtrise rédigé par l'auteur principal, et soumis au Département de sociologie de l'Université de Montréal. La collecte de données initiale a été financée par le ministère des Communautés culturelles et de 1'Immigration du Québec et par 1'Institut québécois de recherche sur la culture. 
contraire ou, plus précisément, certaines études empiriques ne signalent aucune trace évidente de l'effet de la compétence linguistique sur la situation des immigrants eu égard au marché du travail (Portes et Stepick, 1985; Rosenstein, 1985; Evans, 1987; Evans et Kelley, 1987; Renaud, 1992; Goza et al., 1996).

Les écrits recensés ne reposent pas toujours sur des preuves empiriques solides de l'effet des compétences linguistiques. Bien que nous ayons souvent observé ce phénomène, il n'en est pas moins difficile à accepter étant donné que la langue structure nettement les relations sociales. La compétence linguistique a peut-être un effet, mais les études faites jusqu'ici l'ont insuffisamment démontré. Cinq difficultés peuvent expliquer ces observations du non-effet de la langue ou de son effet incertain.

\section{HYPOTHÈSES}

Premièrement, la plupart des études analysent l'effet de la langue sur les revenus et le statut d'un emploi afin de déceler un signe de sa rentabilité. Mais le processus de base qui est à l'œuvre, implicite dans la plupart des études, est que la langue permet l'accès au marché du travail et à la mobilité professionnelle, qui, en retour, permet la mobilité sociale, effet qui peut être vérifié dans les revenus et le statut. Le raisonnement est correct mais indirect. Avant d'obtenir un emploi rémunérateur et de bon statut, encore faut-il avoir un emploi et le conserver. Nous étudions ici le processus de base qu'est la mobilité professionnelle : si la langue a un rôle, c'est sur la vitesse d'accès aux emplois et sur la durée de séjour dans ces emplois qu'il s'exercerait.

Deuxièmement, la plupart des études ont été réalisées dans des pays unilingues (États-Unis et Australie) ou dans des pays bilingues où la compétence dans une seule langue a été prise en considération (Toronto). Dans ces conditions, l'effet de la compétence linguistique et l'effet de l'intégration sociale, bien que logiquement tout à fait différents, ne peuvent pas être distingués nettement l'un de l'autre, étant donné leurs interactions dynamiques dans les relations sociales structurées autour de la langue, c'est-à-dire dans les communautés linguistiques. Nous cherchons à montrer l'effet différencié de ces deux dimensions. Pour ce faire, nous étudions l'établissement des immigrants dans une région bilingue où l'immigration représente une portion significative de la population (19 pour cent 
en 1996). Dans un tel contexte, maîtrise des langues et participation à une communauté linguistique ne sont pas synonymes.

Troisièmement, la plupart des études ont été faites sur les populations immigrantes vivant dans une région donnée, souvent sans autre distinction. Dans ces conditions, les effets reliés à l'histoire (arrivée d'immigrants provenant de pays différents selon la période, climats de crise économique ou de prospérité, etc.), à l'âge et aux différentes durées de séjour, etc. peuvent être confondus. L'utilisation de classements d'immigrants selon l'année d'établissement ne résout le problème qu'en partie, à moins que ce ne soit possible de réduire à moins d'un an l'écart entre l'arrivée du premier et celle du dernier groupe. Autrement, comme les immigrants, même au sein d'un même "groupe d'arrivée ", ont eu des périodes de temps notablement différentes pour apprendre la langue, il serait difficile de savoir ce que l'on mesure exactement. Pour contourner cette difficulté, nous étudions ici un échantillon d'immigrants arrivés à quelques mois d'intervalle.

Quatrièmement, de nombreuses études ne portent que sur des immigrants qui travaillent. Par conséquent, il est impossible de distinguer ce qui favorise ou défavorise la rapidité d'accès ou de sortie des emplois. Comme nous cherchons à examiner l'effet des langues sur la durée des trois premiers épisodes d'emploi et de chômage, nous retiendrons pour nos analyses l'ensemble des immigrants de la cohorte étudiée, peu importe qu'ils soient ou non à la recherche d'un emploi.

Enfin, et ceci est la caractéristique essentielle de notre démarche, l'établissement en emploi est un processus dynamique. Le cheminement professionnel des immigrants n'est jamais fixé pour toujours et par conséquent il ne peut être étudié pleinement de façon statique. C'est un phénomène dynamique qui relève de nombreux facteurs (scolarité, expérience, connaissance des langues, intégration dans des réseaux, etc.). La majorité des études définissent mal ou de façon trop restrictive la notion d'accès au marché du travail. Notre compréhension de l'établissement professionnel des immigrants demeurerait en effet limitée si, comme dans la majorité des études recensées, nous ne nous intéressions qu'à l'effet de prédicteurs sur un premier emploi ou sur le fait de détenir un emploi après un certain laps de temps. Il est possible que l'effet de la compétence linguistique ne reste pas le même durant toute la période d'établissement, qu'il varie en fonction du temps passé dans le pays d'accueil. D'une part, on peut imaginer, par exemple, que 
la maîtrise des langues augmente la mobilité professionnelle à un moment donné et en stimule la stabilisation à un autre. Dès lors, la maîtrise des langues agirait dans des directions opposées selon le moment de la période d'établissement, juste au début ou plus tard. D'autre part, on peut aussi croire que les modalités d'action de la langue changent avec le temps. Au premier contact avec la nouvelle société, la présence ou l'absence de compétence linguistique est essentielle, bien que, plus tard, on s'attende à ce que les réseaux sociaux structurés autour de la langue soient efficients, et non pas seulement la compétence linguistique. Dans notre étude, afin de vérifier si les effets de la langue sont opposés en fonction du moment pris en considération, nous distinguons les emplois en fonction de leur rang (depuis le moment de l'arrivée). Afin de voir à l'œuvre les dimensions " compétence " et "réseaux sociaux reliés à la langue ", nous introduisons, en deçà de la mesure de la compétence linguistique, une mesure de la langue des emplois déjà occupés dans le pays d'accueil.

\section{LE CONTEXTE LINGUISTIQUE : MONTRÉAL, LE QUÉBEC, LE CANADA}

Montréal est la métropole économique de la province de Québec. Au recensement de $1996{ }^{1}$, la population de sa région métropolitaine était de 3,3 millions d'habitants. Le Québec (7,1 millions d'habitants en 1996) est la seule province canadienne à majorité francophone. En 1996, 83,6 pour cent de sa population parlait le français à la maison, 11,6 pour cent l'anglais et 4, 8 pour cent une autre langue. Ces proportions ne s'appliquent pas à la région métropolitaine, qui est le lieu traditionnel de concentration de la communauté anglophone du Québec et, de plus, le lieu privilégié d'établissement des immigrants : 88 pour cent des immigrants du Québec y vivaient en 1996. À cause de cette situation, la région de Montréal contient une plus petite proportion de francophones (70 pour cent) que la province, et une plus grande proportion d'anglophones (19 pour cent) et d'allophones (11 pour cent). En dépit de cela, les francophones constituent la majorité, et leur présence relative s'est accentuée au détriment des anglophones: entre 1971 et 1986, la proportion de francophones est passée de

Source : Statistique Canada, Recensement de 1996, Le Canada en statistiques. Tableau de la série "Le Pays". 
TABLEAU 1 - Utilisation du français au travail en 1989 selon la langue maternelle

\begin{tabular}{lrrrr}
\hline $\begin{array}{l}\text { Temps de travail } \\
\text { en français }\end{array}$ & Total & Français & $\begin{array}{c}\text { Langue maternelle } \\
\text { Anglais }\end{array}$ & Autre \\
\hline $90 \%$ et plus & $51 \%$ & $63 \%$ & $8 \%$ & $24 \%$ \\
$50 \%-89 \%$ & $32 \%$ & $30 \%$ & $37 \%$ & $39 \%$ \\
$49 \%$ et moins & $17 \%$ & $6 \%$ & $55 \%$ & $37 \%$ \\
Total & $100 \%$ & $100 \%$ & $100 \%$ & $100 \%$ \\
N & 3211 & 2389 & 432 & $390 \%$ \\
\hline
\end{tabular}

Source : Béland, 1991, table II.3.

66,3 à 69,3 pour cent, et la proportion d'anglophones de 24,9 à 20,3 pour cent.

Cette division linguistique de Montréal est reflétée dans la langue du travail. Le tableau 1 montre que le français est la langue dominante dans le marché de l'emploi, son usage variant en fonction de la langue maternelle. Plus spécifiquement, on peut noter que les individus qui n'ont ni le français ni l'anglais comme langue maternelle (les immigrants ou leurs enfants) se trouvent dans une situation intermédiaire entre les deux groupes dominants et utilisent le français plus de la moitié du temps.

On observe le même phénomène dans la cohorte d'immigrants étudiée ici (enquête ENI : Établissement des nouveaux immigrants, 1989-1999). Si on laisse de côté les toutes premières semaines d'établissement au Québec, pendant lesquelles l'anglais est plus présent, le français est la langue le plus souvent utilisée dans les emplois occupés par les immigrants (taux moyen de 56 pour cent pour les 140 premières semaines d'observation). De son côté, l'anglais est utilisé dans environ 32 pour cent des emplois, tandis qu'une autre langue prédomine dans 12 pour cent des cas seulement (Renaud et al., $1993: 47)$.

Malgré l'importance numérique du français dans le marché du travail, la compétence en anglais, aussi bien que l'appartenance à la communauté anglophone, tend à procurer des emplois mieux rémunérés et de statut plus élevé. Bien que cette tendance soit en décroissance depuis 1970, elle est cependant toujours présente (tableau 2). Durant la première année de séjour de la cohorte étudiée, en 1990, les unilingues francophones composent la catégorie des moins bien rémuné- 
TABLEAU 2 - Revenu des groupes linguistiques relativement aux francophones unilingues, ajusté selon certaines caractéristiques a, hommes, 15 à 64 ans, travaillant à plein temps, sans fréquentation scolaire, natifs, Montréal métropolitain, 1990

\begin{tabular}{lrrr}
\hline Groupe linguistique & 1970 & 1980 & 1990 \\
\hline Francophones unilingues & 100 & 100 & 100 \\
Francophones bilingues & 108 & 105 & 107 \\
Anglophones unilingues et bilingues & 116 & 106 & 103 \\
\hline
\end{tabular}

Source : Le Français langue commune: enjeu de la société québécoise. Rapport du comité interministériel sur la situation de la langue française, Québec, Les Publications du Québec, 1996, xii + 319 p., tableau 2.8.

a. Scolarité, état matrimonial, nombre de semaines travaillées et d'expérience.

rés (100); les francophones bilingues (107) sont dans une meilleure position.

De plus, chez les immigrants de la cohorte étudiée, seule la connaissance de l'anglais est un atout important pour obtenir un revenu d'emploi plus élevé (tableau 3).

Enfin, si on considère non pas la connaissance des langues chez les immigrants mais la langue caractérisant l'emploi, l'utilisation de l'anglais au travail, aussi bien que l'utilisation du bilinguisme français-anglais, est associée à des emplois plus rémunérateurs que ceux où l'on n'utilise que le français (tableau 4), même si le statut socio-économique des emplois français et anglais est identique (mais celui des emplois bilingues demeure plus avantageux) (Renaud et Lebeau, 1993, tableau II, p. 7). Ce qui nous amène à dire qu'à emploi de statut équivalent, les emplois bilingues ou en anglais sont mieux rémunérés que les emplois en français. À ce titre, l'anglais joue un rôle attractif important auprès des immigrants sur la scène du marché de l'emploi. Il reste à vérifier si cet avantage persiste ou

TABLEAU 3 - Régressions des revenus hebdomadaires d'emplois occupés par des immigrants sur la connaissance des langues

\begin{tabular}{lrrrrr}
\hline & B & E. S. & Beta & T & Sign. T \\
\hline Français, anglais & $-118,08$ & 80,60 & $-0,061$ & $-1,455$ & 0,143 \\
Français & 22,96 & 43,36 & 0,018 & 0,530 & 0,597 \\
Anglais & 214,85 & 56,52 & 0,152 & 3,801 & $0,000^{* *}$ \\
(Constante) & 292,48 & 34,47 & & 8,485 & 0,000 \\
\hline
\end{tabular}

** Significatif à $a \leq 0,01$.

$\left(R^{2}=0,014\right)$. 
TABLEAU 4 - Régressions des revenus hebdomadaires d'emplois occupés par des immigrants sur la langue principale de ces emplois ( $n=1227$ emplois)

\begin{tabular}{lrrccc}
\hline & B & E. S. & Beta & T & Sign. T \\
\hline Français, anglais & 39,40 & 10,66 & 0,113 & 0,728 & $0,000^{* *}$ \\
Français & 24,33 & 8,52 & 0,122 & 0,592 & $0,004^{* *}$ \\
Anglais & 40,42 & 9,70 & 0,182 & 0,778 & $0,000^{* *}$ \\
(Constante) & 235,60 & 21,65 & & 6,345 & 0,000 \\
\hline
\end{tabular}

** Significatif à $a \leq 0,01$.

$\left(R^{2}=0,037\right)$.

non lorsqu'il est question de vitesse d'accès au marché du travail et de stabilité en emploi.

L'observation des inégalités entre francophones et anglophones dans le marché du travail et des nombreux transferts d'immigrants vers le groupe anglophone (qui soulèvent la peur d'une minorisation croissante des francophones à l'intérieur de la Confédération canadienne et à l'intérieur du Québec luimême) a conduit, depuis le début des années 1970, à une série de mesures législatives. Ces lois portent en partie sur la langue d'enseignement : mis à part quelques exceptions, les enfants d'immigrants doivent fréquenter l'école française. Ces lois portent aussi sur la francisation du travail et des entreprises. Les entreprises de plus de 50 employés sont soumises à un processus formel pour obtenir leur sceau de francisation; entre autres éléments, la fonction de traduction, si elle doit être présente, doit s'effectuer au niveau le plus élevé de la hiérarchie afin que l'absence de compétence en anglais ne soit pas un obstacle à l'emploi pour les francophones à quelque niveau que ce soit. Ces lois ont visé en premier lieu l'organisation des grandes entreprises et non celle des petites, mais ces dernières sont néanmoins touchées par des mesures générales relatives, notamment, au droit de tout francophone de travailler dans sa langue, à la normalisation du français comme langue commune ou encore à l'affichage. Ces dispositions ne sont pas orientees vers l'usage individuel de la langue, chacun étant libre d'utiliser la langue de son choix. Il est possible en fait de vivre entièrement en français ou entièrement en anglais à Montréal. Les nouveaux arrivants qui entrent dans le marché de l'emploi peuvent choisir de maîtriser la langue française ou la langue anglaise. D'un emploi à l'autre, ils peuvent aussi s'intégrer à la communauté francophone ou à la communauté anglophone. 


\section{DONNÉES ET MÉTHODES}

\section{Les données}

Les données proviennent des entrevues réalisées pendant les trois premières années de l'enquête ENI. Le but de cette étude est de suivre pas à pas, à l'aide de données datées, divers aspects de l'établissement des immigrants admis au Québec en 1989. Le questionnaire est conçu pour étudier la dynamique du processus. La datation hebdomadaire des principales activités qui lui sont reliées (travail, études, habitation, etc.) permet de suivre une cohorte d'immigrants afin de mieux comprendre les dynamiques qui s'enclenchent au fil du temps et influencent leur établissement, notamment en emploi. Comme les événements sont datés les uns par rapport aux autres, on peut les mettre en relation sans expliquer le passé par le présent.

Parmi les 9645 immigrants de 18 ans et plus qui sont arrivés au Canada entre juillet et novembre 1989 et avaient choisi le Québec comme province de destination, 1867 ont consenti à participer à l'étude. Au moment de la première entrevue (entre juin et août 1989), un échantillon de 1000 d'entre eux vivant dans la région du Montréal métropolitain a été appelé à remplir le questionnaire d'enquête ${ }^{2}$. Cet échantillon est représentatif des immigrants qui sont arrivés au Québec visa en main durant cette période ${ }^{3}$. Pour réduire le plus possible tout biais occasionné par le manque de compétence en français ou en anglais (les deux langues officielles du Canada), les entrevues ont été réalisées face à face en 24 langues différentes (sans compter les variations dialectales).

Les résultats des analyses de la qualité de l'échantillon indiquent que cet échantillon est bien représentatif des immigrants âgés de 18 ans et plus arrivés entre la mi-juin 1989 et novembre 1989 avec un visa obtenu à l'étranger, et admis dans l'une ou l'autre des trois catégories d'immigration (réfugié, indépendant et famille). L'année suivante, soit deux ans après leur arrivée, 271 immigrants n'ont pas répondu au questionnaire du second passage. Selon les analyses effectuées, la déperdition observée au temps 2 influence peu la qualité de

2 Parmi les 1000 répondants du début, 729 ont rempli le deuxième questionnaire d'entrevue et 528 le troisième.

3 Pour une description plus détaillée de l'échantillon, voir Renaud et al., 1992; l'annexe 4 présente les procédures d'échantillonnage. Sur l'évolution de l'échantillon pendant la période des entrevues, voir les annexes dans Renaud, Desrosiers et Carpentier, 1993. 
l'échantillon. Les légers changements observés dans sa composition refléteraient une redéfinition de la population étudiée; dans la mesure où on s'intéresse aux immigrants demeurant encore au Québec, nous ne sommes pas en présence d'un biais échantillonnal. Au troisième passage, 221 immigrants supplémentaires n'ont pas participé à l'enquête. On constate de légers changements dans l'échantillon du temps 3 relativement à celui du temps précédent. Nous sommes probablement face à un début de biais, mais également d'effets qui indiqueraient une redéfinition dynamique de la population étudiée, une partie de celle-ci ayant quitté le Québec avant le troisième passage. Enfin, lors du quatrième passage, réalisé après dix ans de séjour, mais dont les données ne sont pas utilisées ici, on aura réussi à récupérer 171 répondants qui, soit n'avaient pas été retracés, soit avaient refusé de répondre au temps 2 ou au temps 3 . Il est donc raisonnable de concevoir cet échantillon comme représentant, à chaque passage d'observation, la population des immigrants demeurant toujours au Québec.

\section{Les variables}

Les variables dépendantes (six durées: trois périodes de non-emploi et trois périodes d'emploi)

Seuls les trois premiers épisodes d'emploi ont été retenus, car ils couvrent à eux seuls 95 pour cent des épisodes d'emploi vécus par les immigrants de la cohorte pendant leurs trois premières années d'établissement. Nous allons étudier la durée des trois premiers épisodes d'emploi et la durée des trois premiers épisodes de non-emploi. En ce qui concerne celle-ci, nous parlerons de la vitesse d'accès en emploi, une courte période de non-emploi étant synonyme d'accès rapide à un emploi. À propos de la durée des épisodes d'emploi, nous parlerons plutôt de stabilité en emploi.

Les variables sont élaborées sur les dates de début et de fin des épisodes d'emploi. L'accès à un premier emploi correspond au temps écoulé entre l'arrivée au Québec et le début du premier épisode d'emploi. Pour l'accès au second et au troisième épisode d'emploi, la façon la plus valable ${ }^{4}$ de modéliser le

$4 \quad$ Les autres possibilités consistent à prendre le temps écoulé entre l'arrivée au Québec et chaque épisode d'emploi ou le temps écoulé entre le début d'un épisode d'emploi et le début de l'épisode précédent. Étant donné la convergence des résultats, nous avons opté pour la solution qui reflète le plus fidèlement la période d'attente précédant l'occupation d'un emploi. 
temps est de considérer le temps passé sans travail avant chacun d'eux. La durée d'un épisode d'emploi correspond au temps écoulé entre son début et sa fin. Les épisodes qui étaient en cours au moment de la dernière entrevue ont été tronqués au moment de l'entrevue, c'est-à-dire qu'ils sont dans le groupe à risque jusqu'à la sortie d'observation.

Les variables indépendantes

— La maîtrise du français et de l'anglais

Les indicateurs linguistiques utilisés dans cette analyse sont des construits. Ils ont été élaborés à partir de l'information contenue dans le visa d'immigration, saisie à l'arrivée au Canada. Ils synthétisent l'information disponible sur la capacité du répondant de parler français et anglais lors de son arrivée au Québec. Ce sont des variables logiques ou dichotomiques. À leur arrivée au Québec, 55,4 pour cent des répondants de l'échantillon avaient des compétences en français et 28,2 pour cent en anglais.

Si ces indicateurs mesurent adéquatement l'état des compétences linguistiques à un moment précis, ces compétences évoluent, spécialement chez ceux qui ne les avaient pas au début. Il est possible, en fonction de la variation de l'aptitude des immigrants à acquérir une langue, de sous-estimer l'effet de la compétence linguistique dans l'obtention d'emplois ultérieurs. Cependant, puisque 80 pour cent des trois premiers épisodes d'emploi des immigrants ont commencé pendant les 52 semaines suivant leur arrivée, cette sous-estimation ne va probablement pas se révéler très significative. Dans l'enquête, la compétence linguistique a été mesurée à nouveau à chaque entrevue annuelle, mais puisque les changements représentés par ces échelles ne peuvent être datés avec précision, elles ne peuvent être utilisées dans cette analyse.

\section{- La langue du travail}

Mesurer la langue du travail n'est pas chose facile. Son utilisation varie selon l'interlocuteur (supérieur, collègue, subordonné, client ou fournisseur), l'intensité et le niveau (parlé, écrit) de l'interaction et la nature du travail (le travail des traducteurs, par exemple, est presque entièrement défini par la langue tandis que, dans la définition d'emplois impliquant des tâches manuelles répétitives, la langue compte peu). Pour les 
besoins de cette analyse, nous avons utilisé la perception des répondants pour repérer "la langue principale utilisée au travail ". Ce choix va de pair avec notre intention de mesurer "le réseau social construit autour de la langue".

La langue du travail peut être observée emploi par emploi ou peut être établie comme une séquence des langues utilisées dans les emplois successifs. La première méthode reflète de plusieurs façons la communauté linguistique à laquelle l'emploi " appartient". La séquence des langues utilisées dans les emplois occupés fournit un indicateur de l'instauration d'une adhésion à une communauté linguistique. Ainsi, au troisième emploi, les séquences "français-français-français " et " anglaisanglais-anglais " caractérisent des individus qui circulent aisément et librement dans les institutions d'une communauté linguistique donnée; nous pouvons imaginer qu'ils y sont socialement intégrés. Au contraire, les répondants dont les séquences comportent le passage d'une langue à une autre au travail (français-anglais-autre, français-anglais-français, etc.) ne s'intégreraient pas socialement, par le travail, à une communauté linguistique. Afin de mieux comprendre l'impact éventuel de ces séquences d'emploi, nous examinerons la mobilité entre langues d'emploi afin de voir si les orientations données par les emplois successifs tendent ou non à se cumuler, à se confirmer, au fil du temps. Comme nous l'avons mentionné, connaissant les dates de début et de fin de chacun des épisodes d'emploi des immigrants, ainsi que la langue la plus couramment utilisée dans chacun d'eux, il nous est possible de reconstituer les cheminements entre les langues d'emploi.

Le point de départ de l'observation est l'arrivée au Québec et les transitions examinées sont l'accès aux trois premiers emplois, selon la langue de ces derniers. Évidemment, les possibilités de transitions vers un emploi dans une langue donnée se multiplient autant de fois qu'il y a de langues considérées. Par exemple, une personne accédant à un premier emploi en français peut par la suite se diriger directement vers un deuxième emploi en français ou bien se diriger vers un deuxième emploi en anglais ou encore vers un emploi dans autre langue. Elle peut évidemment garder le même emploi ou encore le quitter ou le perdre et ne pas réintégrer ensuite le marché du travail.

La figure 1 présente chacune des transitions possibles entre langues d'emploi, avec les probabilités et les effectifs de départ qui y sont associés. Ces effectifs constituent l'échantillon de départ pour le calcul des transitions subséquentes. 
Comme nous travaillons sur une fenêtre de trois ans, les probabilités sont estimées 140 semaines après le début de chaque état. Afin de ne pas commenter des probabilités de transition non crédibles statistiquement, nous laisserons de côté les transitions calculées sur des effectifs de moins de 20 personnes. La somme des transitions partant d'un noud est de un, et les flèches pointillées représentent en quelque sorte les immigrants de la cohorte étudiée qui n'ont pas connu de changement sur la période d'observation de 140 semaines. On notera enfin que les effectifs d'un nœud ne sont pas simplement ceux du nœud précédent multipliés par la probabilité d'y transiter puisqu'il y a des sorties d'observation (cas tronqués).

Si on observe les probabilités d'effectuer chacune des transitions possibles à l'arrivée, on remarque que les chances d'accéder à un premier emploi en français ( 46 pour cent) sont deux fois plus élevées que celles d'accéder à un premier emploi en anglais (23 pour cent), et plus de trois fois plus que celles d'accéder à un premier emploi dans une autre langue (13 pour cent). On a donc tort de croire que les immigrants se dirigent massivement dès le départ vers des emplois où ils travailleront en anglais ou dans une autre langue. Plus encore, les résultats qui suivent montrent que l'attrait du français se maintient également pour les autres périodes d'emploi.

En ce qui concerne le rôle dynamique de la langue, il faut remarquer que les séquences homogènes se confirment. Le fait d'avoir traversé deux périodes consécutives d'emploi en français semble éliminer, pratiquement, les chances d'aller vers un troisième emploi en anglais ou dans une autre langue. Les probabilités de transition sont alors de 60 pour cent pour le français, de 4 pour cent pour l'anglais et de 2 pour cent pour une autre langue. On peut penser que les immigrants qui ont traversé cette séquence "appartiennent", sur le plan du marché du travail, au groupe francophone pour le reste de leur établissement. De façon similaire, le fait d'avoir eu deux emplois en anglais renforce la probabilité d'un troisième emploi dans cette langue, mais de façon légèrement moins marquée que pour le français (50 pour cent).

Dans le cas d'un premier emploi dans une langue autre que le français ou l'anglais, l'avenir linguistique au travail demeure ouvert, les trois langues exerçant un attrait quasi égal (français, 17 pour cent; anglais, 21 pour cent; autre langue, 21 pour cent). L'accès à un premier emploi dans une autre langue ouvre donc toutes les possibilités. Plus précisément, en 


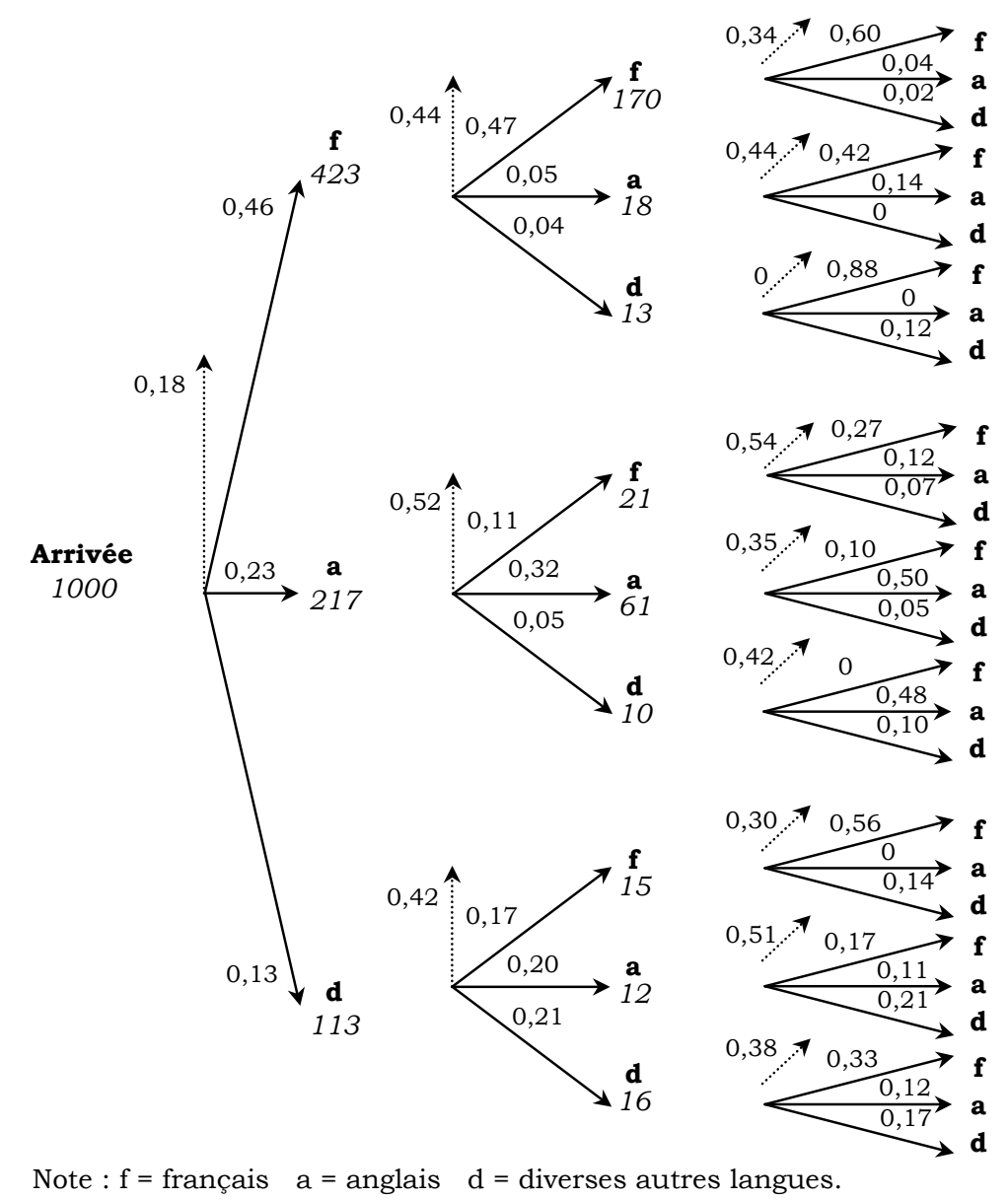

FIGURE 1 - Arborescence des transitions entre langues d'emploi

donnant des chances égales à chacune des trois, ce circuit réduit les chances d'aller vers le français. On peut penser que ces emplois créent une relation entre leurs titulaires et les cohortes d'immigration plus anciennes et leurs orientations linguistiques. L'accès à toute langue sur le marché de l'emploi demeure possible, mais le français perd son rôle prépondérant.

Des déplacements successifs dans des emplois de langues différentes annulent cet effet. Si un premier emploi en anglais est suivi d'un second en français ou si un premier emploi en français est suivi d'un emploi en anglais, il y a une " période de prolongation " qui mène à un match nul par rapport au départ. 
Les contextes linguistiques des emplois paraissent ainsi jouer un rôle important dans la détermination de la langue des emplois subséquents. Leur rôle évolue en fonction de l'expérience accumulée par les immigrants. En ce qui a trait à la dernière partie des analyses de régression, nous utilisons ces séquences comme prédicteurs de l'obtention d'un emploi et de sa durée. Il est possible que des orientations successives vers des emplois dans une langue donnée influent sur les probabilités d'obtenir et de garder un emploi, sans égard aux habiletés linguistiques des personnes impliquées. Si c'est le cas, nous aurons ainsi une mesure de l'effet de l'établissement de contacts avec un des groupes sociaux structurés autour de la langue, les francophones et les anglophones. Il importe donc de départager l'effet des compétences linguistiques et celui des réseaux linguistiques.

\section{Les variables contrôle}

L'âge (en années), la catégorie d'admission (famille, indépendant, réfugié), la scolarité (en années) et l'expérience de travail antérieure à la migration (oui-non) sont généralement considérés comme les clés d'entrée dans le marché du travail. Conséquemment, on doit les contrôler afin de mesurer adéquatement l'effet net des indicateurs linguistiques, qui sont au centre de notre analyse. À des fins descriptives, il est utile de noter que les immigrants de l'échantillon avaient déjà terminé 13 ans de scolarité en moyenne avant leur arrivée et que 78 pour cent d'entre eux avaient une expérience de travail prémigratoire. L'âge moyen des immigrants de l'échantillon est d'environ 35 ans; les hommes y comptent pour 56 pour cent, les immigrants admis comme "indépendants " 5 pour 69 pour cent, et les immigrants appartenant à la catégorie de la "famille " 6 pour 20 pour cent; les autres sont des réfugiés ${ }^{7}$.

5 La candidature des indépendants est évaluée en fonction d'une grille de sélection fondée, en particulier, sur le niveau de scolarité, une préparation professionnelle spécifique, l'employabilité, la demande dans des emplois recherchés, l'expérience professionnelle, l'âge, la connaissance du français et de l'anglais et, enfin, la présence de membres de la famille ou d'amis au Québec.

6 Ces immigrants sont ceux qui ont des liens familiaux avec des citoyens canadiens ou des résidents permanents vivant au Québec.

$7 \quad \mathrm{Au}$ sens de la Convention de Genève, les réfugiés sont des personnes qui sont dans une situation de détresse telle qu'elles méritent une aide humanitaire. 
Enfin, pour la partie de notre analyse qui concerne la dissociation de l'effet de la compétence linguistique de l'effet du réseau social construit autour de la langue, nous contrôlons l'expérience de travail (mesurée en semaines) acquise au Québec avant l'événement étudié (l'épisode de recherche d'emploi et l'épisode en emploi). Ce contrôle est nécessaire afin d'éviter d'interpréter l'effet global de l'entrée dans le marché du travail montréalais comme l'effet d'un réseau linguistique donné.

\section{Méthodes}

Étant donné le caractère dynamique de l'établissement de l'immigrant en emploi et des données utilisées, nous allons recourir à des analyses de régression de survie. Nous utilisons une version multivariée particulière de ce modèle qui nous permet de tester directement si la compétence linguistique a des effets différents sur la durée de l'épisode en fonction du rang des épisodes.

Cette première analyse sera réalisée avec des analyses de régression multivariées, du modèle de Cox (plusieurs variables dépendantes simultanément). Nous testerons l'équivalence de coefficients entre les régressions (différentes par la variable dépendante : durée du premier, deuxième et troisième épisode) et, le cas échéant, nous estimerons la valeur commune de ces coefficients. Étant donné que nous cherchons les différents effets des indicateurs linguistiques en fonction du rang de l'épisode d'emploi, nous examinons en premier lieu s'il y a une différence (tests de différence) ou non entre les coefficients de régression. S'il y a une différence significative entre les coefficients, nous ne regarderons que les indicateurs spécifiques pour chacun des épisodes d'emploi. S’il n'y a pas de différence significative, nous examinerons la valeur commune des coefficients, ce qui donnera la meilleure estimation.

Cette méthode ne permet cependant pas d'introduire des variables indépendantes variant dans le temps, ni des variables indépendantes spécifiques à chacune des durées de séjour dans un état donné. Conséquemment, nous reviendrons aux régressions "à risque proportionnel " usuelles quand nous introduirons dans l'analyse des variables différentes pour chaque rang d'emploi ou de non-emploi, sans recourir alors au modèle multivarié. 


\section{RÉSULTATS}

\section{L'inversion de l'effet de la langue}

Les tableaux 5 et 6 donnent les résultats des régressions de survie sur l'accès aux emplois et leur durée. Avant d'examiner l'effet de la compétence linguistique, observons l'effet des variables contrôle sur l'accès à l'emploi (tableau 5). Quatre d'entre elles exercent des effets significatifs différents en fonction du rang de l'épisode d'emploi. Parmi ces quatre variables, seul l'âge tend à diminuer la probabilité que les immigrants accèdent à un premier et à un second emploi, mais cet effet disparaît durant leur intégration au marché du travail. Inversement, les immigrants de sexe masculin et ceux qui ont une expérience de travail antérieure à leur arrivée, aussi bien que les "indépendants " et les immigrants de la catégorie "famille", accèdent plus rapidement à un premier emploi, mais cet avantage disparaît ultérieurement.

TABLEAU 5 - Régressions de survie de l'accès aux trois premiers emplois

\begin{tabular}{|c|c|c|c|c|c|}
\hline \multirow[b]{2}{*}{ Variables } & \multicolumn{3}{|c|}{ Accès à l'emploi de rang... } & \multirow{2}{*}{$\begin{array}{c}\text { Test de } \\
\text { différence }\end{array}$} & \multirow{2}{*}{$\begin{array}{c}\text { Pente } \\
\text { commune }\end{array}$} \\
\hline & $\ldots 1$ & $\ldots 2$ & $\ldots 3$ & & \\
\hline Connaissance du français & 0,010 & $0,440 * *$ & 0,290 & ** & - \\
\hline Connaissance de l'anglais & $-0,040$ & 0,060 & 0,330 & $\begin{array}{c}\mathrm{ns} \\
* *\end{array}$ & ns \\
\hline Âge & $-0,040^{* *}$ & $-0,020^{* *}$ & $-0,008$ & $* *$ & - \\
\hline $\begin{array}{l}\text { Sexe (homme }=1 \text {, } \\
\text { femme }=0 \text { ) }\end{array}$ & $0,500 * *$ & $-0,007$ & 0,280 & ns & - \\
\hline Scolarité & $-0,004$ & 0,003 & $-0,050^{*}$ & $* *$ & ns \\
\hline $\begin{array}{l}\text { Expérience de travail } \\
\text { prémigratoire }\end{array}$ & $0,690 * *$ & 0,190 & 0,080 & & - \\
\hline (Catégorie d'admission) & & & & & \\
\hline $\begin{array}{l}\text { Famille } \\
\text { Indépendant } \\
\text { (réfugié) }\end{array}$ & $\begin{array}{l}0,510^{* *} \\
0,510^{* *}\end{array}$ & $\begin{array}{l}-0,360 \\
-0,340\end{array}$ & $\begin{array}{r}-0,020 \\
-0,020\end{array}$ & $* *$ & - \\
\hline$-2 L L$ & 202,56 & 25,92 & 10,18 & & \\
\hline $\mathrm{N}$ & 974 & 437 & 198 & & \\
\hline Cas tronqués & 227 & 125 & 58 & & \\
\hline
\end{tabular}

* significatif à $a \leq 0,05 ;{ }^{* *}$ significatif à $a \leq 0,01$; ns : non significatif; - : ne s'applique pas. 
De façon plus spécifique à notre propos, seule la maîtrise du français (et non de l'anglais) par les immigrants influence de façon différentielle, d'un rang à l'autre, la probabilité d'accéder aux emplois. Si la maîtrise du français ne semble pas, au début, accélérer l'obtention d'un premier emploi, une fois l'immigrant entré dans le marché du travail, elle diminue l'attente d'un second emploi. La maîtrise de l'anglais n'exerce aucun effet significatif ni ne présente de différences entre les épisodes.

Quand nous observons selon leur rang la durée des épisodes d'emploi comme variables dépendantes (tableau 6), nous remarquons des effets très réduits pour ce qui est des variables contrôlées mais aussi un effet important de la compétence en français. Parmi les variables contrôles, seule la scolarité exerce des effets significatifs différents selon le rang des épisodes d'emploi, mais elle n'a pas d'effets significatifs sur les épisodes d'emploi. Quant aux indicateurs linguistiques, seule la maîtrise du français a, encore une fois, des effets significativement différents (significatifs pour chaque emploi) sur la durée des trois premiers épisodes d'emploi. Le fait de connaitre le français accélère la fin des deux premiers épisodes d'emploi, mais accroît au contraire la stabilité dans le troisième emploi.

TABLEAU 6 - Régressions de survie de la durée des trois premiers épisodes d'emploi

\begin{tabular}{lrrrrr}
\hline & \multicolumn{2}{c}{ Rang de l'emploi } & \multicolumn{2}{c}{ Test de } & Pente \\
Variables & 1 & 2 & 3 & différence commune \\
\hline Connaissance du français & $0,44^{*}$ & $0,400^{*}$ & $-0,660^{*}$ & $* *$ & - \\
Connaissance de l'anglais & $-0,130$ & 0,140 & $-0,430$ & $\mathrm{~ns}$ & $\mathrm{~ns}$ \\
Âge & $-0,080$ & 0,090 & 0,480 & $\mathrm{~ns}$ & $\mathrm{~ns}$ \\
Sexe (H = 1, F =0) & $-0,010^{*}$ & 0,003 & $-0,010$ & $\mathrm{~ns}$ & $\mathrm{~ns}$ \\
Scolarité & $-0,020$ & $-0,020$ & 0,060 & $*$ & - \\
Expér. de travail prémigr. & $-0,020$ & $-0,270$ & $-0,360$ & $\mathrm{~ns}$ & $\mathrm{~ns}$ \\
(Catégorie d'admission) & & & & & \\
Famille & $-0,170$ & $-0,300$ & $-0,100$ & $\mathrm{~ns}$ & $\mathrm{~ns}$ \\
Indépendant & $-0,180$ & $-0,340$ & $-0,030$ & $\mathrm{~ns}$ & $\mathrm{~ns}$ \\
(réfugié) & & & & & \\
- 2LL & 32,06 & 7,17 & 13,81 & & \\
$\mathrm{~N}$ & 743 & 335 & 151 & & \\
Cas tronqués & 306 & 137 & 81 & & \\
\hline
\end{tabular}

* significatif à $a \leq 0,05 .{ }^{* *}$ significatif à $a \leq 0,01$. ns : non significatif. - : ne s'applique pas. 
Toutes choses étant égales, la maîtrise du français joue un rôle important dans la mobilité professionnelle des immigrants. Nous supposions au départ que l'influence de la compétence linguistique de l'immigrant pouvait varier en cours d'établissement, et il existe bien un effet de la compétence linguistique variant avec le temps, selon le rang de l'épisode. La maîtrise du français ne semble pas favoriser systématiquement l'accès à l'emploi au début, mais l'effet positif de la compétence en langue française sur la mobilité professionnelle apparaît par la suite. De plus, l'effet de la compétence en langue française raccourcit la durée de séjour dans un emploi au début et l'allonge plus tard. Lors de l'insertion dans le marché du travail québécois, il semble que le besoin des immigrants de trouver du travail rapidement soit tel que leurs compétences linguistiques n'apparaissent pas, au départ, comme un élément de capital humain. La valeur de cette compétence n'est ressentie qu'à long terme, une fois qu'ils ont obtenu un premier emploi. On peut imaginer qu'après leur entrée dans le marché du travail, l'absence de frontières linguistiques accorderait aux immigrants parlant français la capacité de "magasiner ": ils pourraient alors avoir la chance de trouver un emploi qui leur convient. Pour cette raison, ils tendraient à quitter plus rapidement leurs deux premiers emplois, et la capacité de se stabiliser procurée par la maîtrise du français ne se manifesterait pas avant le troisième emploi. Les résultats indiquent donc que les mécanismes qui mènent à l'obtention d'un emploi réagissent différemment à la compétence en français selon le rang des épisodes ${ }^{8}$. Enfin, constat important, l'anglais ne joue aucun rôle. La maîtrise de l'anglais n'a pas d'effet sur l'accès au marché du travail ou sur la mobilité professionnelle pendant les trois premières années au Québec.

\section{Habiletés linguistiques et réseaux sociaux construits autour de la langue du travail}

Les résultats de la première partie de l'analyse nous ont permis de dégager un certain nombre de constats sur les compétences linguistiques, définies par la connaissance des langues française et anglaise. Mais ces résultats ne tiennent pas compte

8 D'autres analyses, qui ne sont pas présentées dans cet article, ont montré que c'est le rang des épisodes d'emploi des immigrants qui est réellement en cause et non le fait d'avoir eu un épisode d'emploi dans une période de temps donnée (première année, six premiers mois, etc.). 
d'une autre réalité linguistique qui, on l'a vu, est susceptible de varier au fil du temps et est moins directe que la connaissance des langues par les acteurs. C'est pourquoi nous examinerons maintenant si c'est la connaissance de la langue qui est à l'œuvre ou si ce ne seraient pas plutôt les liens tissés avec une communauté linguistique à travers les emplois. Pour ce faire, nous observerons, à l'aide de régressions de survie traditionnelles (modèle de Cox de base), l'effet de la langue des emplois antérieurs à l'épisode étudié sur les durées des épisodes de chômage et d'emploi, en contrôlant par la connaissance des langues. Nous prendrons comme variables indépendantes, non pas l'ensemble des séquences possibles entre langues d'emploi, mais plutôt les séquences homogènes en français et en anglais, qui nous intéressent plus particulièrement. Nous isolerons également la durée (en semaines) de l'expérience de travail accumulée au Québec au début de chacun des épisodes observés pour départager l'effet de l'expérience de travail acquise au Québec et l'effet de la langue.

Si l'effet de la compétence linguistique observé plus haut disparait quand nous introduisons des séquences de travail homogènes linguistiquement, nous pourrons raisonnablement conclure que l'action de la langue est due à la construction d'un lien social avec la communauté linguistique plutôt qu'à un usage fonctionnel de la langue elle-même.

\section{Épisodes d'accès à l'emploi ${ }^{9}$}

La langue du travail, ou plutôt la séquence des langues du travail antérieure à l'épisode examiné, utilisée comme prédicteur de la probabilité d'accès à l'emploi (tableau 7), ne donne pas d'effets nets significatifs statistiquement quand on isole la connaissance linguistique. La construction d'une adhésion à une communauté linguistique, mesurée par la langue des emplois occupés, n'apparaît pas comme un élément déterminant de la vitesse d'acquisition d'un emploi.

Un autre élément important à souligner, par rapport aux résultats présentés au tableau 5, est que l'effet positif de la connaissance du français sur l'accès à un second emploi disparaît quand on introduit les séquences de langues du travail et qu'on isole l'expérience de travail acquise au Québec.

9 Dans cette partie de l'analyse, les séquences de travail aussi bien que la durée (en semaines) de l'expérience de travail acquise au Québec sont rapportées seulement aux épisodes suivant le premier emploi. 
TABLEAU 7 - Régressions de survie sur l'accès aux emplois selon le rang et la langue du travail

\begin{tabular}{lccc}
\hline & \multicolumn{3}{c}{ Accès aux emplois de rang... } \\
Variables & $\ldots 1$ & $\ldots 2$ & $\ldots 3$ \\
\hline Connaissance du français & - & 0,220 & 0,270 \\
Connaissance de l'anglais & - & 0,200 & 0,270 \\
Âge & - & $-0,005$ & $-0,020^{*}$ \\
Sexe (homme = 1, femme = 0) & - & 0,150 & 0,090 \\
Scolarité & - & 0,020 & $-0,040$ \\
Expér. de travail prémigratoire & - & 0,230 & $0,500^{*}$ \\
(Catégorie d'admission) & & & \\
Famille & - & $-0,330$ & 0,360 \\
Indépendant & - & $-0,350$ & 0,150 \\
Emploi_1_français & - & 0,310 & - \\
Emploi_1_anglais & - & 0,400 & - \\
Expér. de travail au Québec & - & $-0,020^{* *}$ & $-0,008^{* *}$ \\
Emploi_1 + 2_français & - & - & $-0,230$ \\
Emploi_1 + 2_anglais & - & - & 0,040 \\
- 2LL & - & 90,34 & 19,01 \\
N & - & 431 & 151 \\
Cas tronqués & - & 213 & 81 \\
\hline
\end{tabular}

* significatif à $a \leq 0,05, * *$ significatif à $a \leq 0,01$.

Le seul déterminant statistiquement significatif de l'accès à un second emploi qui apparaisse ici est la durée de l'expérience de travail acquise au Québec avant le deuxième emploi, et son effet est négatif. Ainsi, plus un immigrant accumule de semaines d'expérience de travail dans un premier emploi, moins il y a de chances qu'il aille rapidement vers un deuxième emploi. Comme il s'agit du seul élément significatif, on doit comprendre que l'étendue de l'expérience de travail acquise au Québec se trouve à médiatiser l'effet de la connaissance des langues que nous avions décelé précédemment (voir le tableau 5).

Parmi les variables contrôles présentant un effet significatif sur l'accès à un troisième emploi figurent l'âge, l'expérience de travail au Québec et l'expérience de travail antérieure à la migration. Ainsi, contrairement à ce que nous avons vu dans la première partie de l'analyse, quand on isole la langue du travail 
et la durée de l'expérience de travail acquise au Québec, on constate que la présence d'une expérience de travail antérieure à la migration est un capital humain qui influence l'accession à un troisième emploi. Les immigrants qui ont ce type d'expérience de travail ont deux fois et demie $\left(e^{0,5}=1,6\right)$ plus de chances d'accéder plus rapidement à un troisième emploi que ceux qui en sont dépourvus. Ces effets montrent en partie que le troisième emploi est plus "recherché ", plus " convoité ", en ce sens que les individus doivent posséder des caractéristiques " avantageuses" pour l'obtenir plus rapidement, c'est-à-dire être jeunes et avoir de l'expérience professionnelle avant leur arrivée au Québec.

Enfin, même si la langue du travail est représentée (comme langue de chaque épisode précédant celui qui est étudié ou comme séquence des langues du travail), elle n'a pas d'effet net statistiquement significatif quand on isole la compétence linguistique, qui perd ici l'effet positif décelé précédemment (voir le tableau 5). Toutes choses étant égales, la construction d'une adhésion à une communauté linguistique (par l'utilisation de sa langue au travail) ne semble pas jouer de rôle déterminant dans la vitesse à laquelle un emploi est obtenu.

\section{La stabilité en emploi}

Rappelons que la compétence en langue française raccourcit la durée du premier et du second emploi et allonge celle du troisième (voir le tableau 6). Le tableau 8 montre que, quand on introduit seulement la langue de l'emploi en cours, on n'observe pas d'effet significatif sur la durée des deux premiers emplois. Cependant, quand des séquences de langues homogènes sont introduites, l'effet de la compétence en langue française sur la durée du troisième emploi disparaît au profit de l'effet de cette variable. Les langues du travail ne jouent un rôle que lorsque le troisième emploi et les deux précédents sont majoritairement en français ${ }^{10}$. Dans la durée de l'épisode du troisième emploi, ce n'est pas la compétence en langue française qui favorise la stabilité, bien qu'elle ait son propre effet pendant les deux premiers épisodes (voir le tableau 6), mais plutôt le réseau social construit autour de la langue de travail.

10 Nous avons aussi obtenu un effet significatif pour les séquences emploi_1français + emploi_2-français et emploi_2-français + emploi_3-français. Cependant, si nous enlevons de ces paires celles qui font partie des séquences de trois épisodes, les coefficients ne sont plus significatifs. C'est donc la séquence des trois emplois en langue française qui est à l'œuvre. 
TABLEAU 8 - Régressions de survie pour la durée des emplois selon le rang et la langue du travail

\begin{tabular}{|c|c|c|c|}
\hline \multirow[b]{2}{*}{ Variables } & \multicolumn{3}{|c|}{ Rang de l'emploi } \\
\hline & 1 & 2 & 3 \\
\hline Connaissance du français & $0,450^{* *}$ & 0,210 & $-0,540$ \\
\hline Connaissance de l'anglais & $-0,060$ & 0,100 & $-0,560$ \\
\hline Âge & $-0,010^{*}$ & 0,000 & $-0,010$ \\
\hline Sexe $($ homme $=1$, femme $=0$ ) & $-0,090$ & 0,150 & 0,360 \\
\hline Scolarité & $-0,020$ & $-0,020$ & $-0,080^{*}$ \\
\hline Expér. de travail prémigratoire & 0,010 & $-0,300$ & $-0,260$ \\
\hline \multicolumn{4}{|l|}{ (Catégorie d'admission) } \\
\hline Famille & $-0,130$ & $-0,400$ & $-0,000$ \\
\hline Indépendant & $-0,150$ & $-0,410$ & 0,020 \\
\hline Emploi_1_français & $-0,160$ & - & - \\
\hline Emploi_1_anglais & 0,300 & - & - \\
\hline Expérience de travail au Québec & - & $-0,010^{* *}$ & $-0,020 * *$ \\
\hline Emploi_1 + 2_français & - & 0,340 & - \\
\hline Emploi_1 + 2_anglais & - & 0,280 & - \\
\hline Emploi_1 $+2+3$ _français & - & - & $-0,810^{* *}$ \\
\hline Emploi_1 + 2 + 3_anglais & - & - & $-0,360$ \\
\hline$-2 L L$ & 164,79 & 2023,52 & 571,09 \\
\hline $\mathrm{N}$ & 731 & 335 & 571,09 \\
\hline Cas tronqués & 300 & 137 & 81 \\
\hline
\end{tabular}

* significatif à $\mathrm{a} \leq 0,05, * *$ significatif à $\mathrm{a} \leq 0,01$.

\section{CONCLUSION}

Quel que soit l'angle sous lequel on examine ces questions, l'impact de la langue se modifie ou varie tout au long de la période d'établissement. Nous avons vu dans un premier temps que la connaissance du français, qui n'est pourtant d'aucun secours pour obtenir un premier emploi, toutes choses étant égales, augmente la circulation entre les emplois pendant le début de l'établissement pour permettre d'atteindre subséquemment la stabilisation en emploi. En observant les langues du travail plutôt que les habiletés linguistiques des immigrants, nous avons aussi vu qu'un déplacement vers un nouvel emploi dépendait en bonne partie de la séquence des langues des emplois occupés au Québec. 
Globalement donc, la langue française semble avoir un effet sur la mobilité professionnelle. Elle rend les immigrants plus mobiles sur le marché du travail. Sur une fenêtre d'observation de trois ans suivant leur arrivée au Québec, elle leur permet de quitter leurs deux premiers emplois plus rapidement et de conserver un troisième emploi plus longtemps. Bien que la connaissance du français en soi semble responsable de l'action de la langue au début de l'établissement, ce sont les contacts professionnels avec la communauté francophone (que cette connaissance permet) qui agissent ultérieurement.

Même si on pouvait penser que l'anglais et le français avaient une influence semblable partout, ce n'est pas le cas. Seul le français joue un rôle ici. La compétence en langue anglaise et son utilisation au travail n'ont pas d'impact sur la mobilité professionnelle des immigrants. Le plus étonnant de tout cela est que, traditionnellement, ces derniers se sont intégrés massivement à la communauté anglophone. Serait-ce un effet récent de la législation sur la langue au Québec, qui a contraint les grandes entreprises à adopter le français ? $\mathrm{Si}$, dans le contexte canadien global, l'anglais a nettement une position de langue majoritaire, elle aurait perdu ce statut à Montréal. D'autres comparaisons sont nécessaires avant que l'on soit en mesure d'avancer des explications solides. Provisoirement, nous pouvons peut-être supposer que la langue qui est " efficace " est celle de la majorité locale, celle de Montréal et du Québec, et que la "carrière linguistique au travail " semble être un important facteur de l'intégration à une communauté linguistique donnée. D’autres études ont déjà montré le rôle des communautés ethniques dans l'établissement en emploi des immigrants; celle-ci aura à tout le moins montré l'importance de la prise de contact avec le groupe majoritaire local, qui constitue la principale communauté d'accueil.

La langue n'agit pas de façon monotone; elle n'a pas un impact uniforme. Son impact dépend de la progression de l'établissement et des expériences de travail. La langue est autant une question de contexte d'accueil que de connaissance par les individus.

Sur un autre plan, il reste à vérifier si le modèle d'évolution de l'impact de la langue que nous avons observé est valable après le troisième emploi. Nous venons de terminer la quatrième vague d'observation de cette cohorte, portant à dix années de séjour au Québec la fenêtre d'observation (Renaud et al., 2001). Il nous faut déjà reprendre et poursuivre ces analyses. 


\section{RÉFÉRENCES BIBLIOGRAPHIQUES}

AMISSAH, C. K. 1996. "Sub-Saharan Africans in the US labor market: The cost of being Black ", National Journal of Sociology, 10, 1 : 5782 .

BÉLAND, P. 1991. L'Usage du français au travail. Situation et tendances. Gouvernement du Québec, Conseil de la langue française, Dossiers, no 33.

BLOOM, D. E., et G. GRENIER. 1996. "Language, employment, and earnings in the United States: Spanish-English differentials from 1970 to 1990 ", International Journal of the Sociology of Language, 121 : 45-68.

DRINKWATER, S. J., et N. C. O'LEARY. 1997. "Unemployment in Wales: Does language matter? ", Regional Studies, 31, 6 : 583-591.

EVANS, M. D. R. 1987. "Language skill, language usage and opportunity: Immigrant in the Australian labour market ", Sociology, 21, 2 (mai) : 253-274.

EVANS, M. D. R., et J. KELLEY. 1986. "Immigrants' work: Equality and discrimination in the Australian labour market ", Australian and New Zealand Journal of Sociology, 22, 2 : 187-207.

GOZA, F., V. LUSE et A. DEMARIS. 1996. Unemployment Transitions Among Brazilian Immigrants in the United States and Canada. Ohio, Bowling Green State University, Department of Sociology, Association Paper, $24 \mathrm{p}$.

KOSSOUDJI, S. A., et D. A. COBB-CLARK. 1996. "Finding good opportunities within unauthorized markets. US occupational mobility for male Latino workers ", International Migration Review, 30, 4 : 901-924.

MAJKA, L., et B. MULLAN. 1991. "Employment retention, area of origin and type of social support among refugees in the Chicago area ", International Migration Review, XXVI, 3 : 899-926.

MCALLISTER, I. 1986. "Speaking the language: Language maintenance and English proficiency among immigrant youth in Australia ", Ethnic and Racial Studies, 9, $1: 24-42$.

MILLER, P. W., et L. M. NEO. 1997. "Immigrant unemployment. The Australian experience ", International Migration, 35, 2 : 155-185.

PORTES, A., et A. STEPICK. 1985. "Unwelcome immigrants: The labor market experiences of 1980 (Mariel) Cuban and Haitian refugees in South Florida ", American Sociological Review, 50 : 493-514.

RENAUD, J. 1992. "Un an au Québec. La compétence linguistique et l'accès à un premier emploi ", Sociologie et sociétés, XXIV, 2 : 131142.

RENAUD, J., et R. LEBEAU. 1993. "La langue stratifie-t-elle les emplois des nouveaux immigrants ? ", dans J. RENAUD, S. CRESPO, R. LEBEAU, C. MONTGOMERY et P. GOYETTE. Nouveaux immi- 
grants et langue. Université de Montréal, Département de sociologie, Rapport de recherche soumis à l'Office de la langue française.

RENAUD, J., A. CARPENTIER, G. OUIMET et C. MONTGOMERY. 1992. La Première Année d'établissement d'immigrants admis au Québec en 1989: Portraits d'un processus. Montréal, Gouvernement du Québec, Ministère des Communautés culturelles et de l'Immigration; http://www.ceetum.umontreal.ca/eni/Portrait_T1.pdf.

RENAUD, J., S. CRESPO, R. LEBEAU, C. MONTGOMERY et P. GOYETTE. 1993. Nouveaux Immigrants et langue. Université de Montréal, Département de sociologie, Rapport de recherche déposé à l'Office de la langue française, $222 \mathrm{p}$.

RENAUD, J., S. DESROSIERS et A. CARPENTIER. 1993. Trois Années d'établissement d'immigrants admis au Québec en 1989. Portraits d'un processus. Montréal, Gouvernement du Québec, Ministère des Communautés culturelles et de l'Immigration; http://www. ceetum.umontreal.ca/eni/Portrait_T3.pdf.

RENAUD, J., L. GINGRAS, S. VACHON, C. BLASER, J.-F. GODIN et B. GAGNÉ. 2001. Ils sont maintenant d'ici! Les dix premières années au Québec des immigrants admis en 1989. Sainte-Foy, Les Publications du Québec, MRCI, collection Études, recherches et statistiques, no 4, xxxvi +197 p., 5 cartes et 350 figures.

ROSENSTEIN, C. N. 1985. The Effects of Immigration on the Occupational Careers of Recent Immigrants to Canada. Los Angeles, University of California, Doctoral Dissertation.

SHU, J. 1996. "Labor force status in Australia of newly arrived immigrants from Asia ", Asian Migrant, 9, $2:$ 47-54.

STOLZENBERG, R. M., et M. TIENDA. 1997. "English proficiency, education, and the conditional economic assimilation of Hispanic and Asian origin men ", Social Science and Research, 26, $1: 25-51$.

\begin{abstract}
Ronald LEBEAU and Jean RENAUD

\section{LANGUAGE AND OCCUPATIONAL MOBILITY IN THE MONTRÉAL LABOUR MARKET FOR IMMIGRANTS ARRIVING IN 1989: A LONGITUDINAL} APPROACH

The economic establishment of new immigrants is often linked to their ability to speak the language of the host country. This simple and intuitive hypothesis now has to be proven, as most studies do not show a relation between language and labour market status. Moreover, a review of the literature does not always provide sound, empirical proof of the effect of language proficiency. This paper takes a new look at this issue. The study focuses on new immigrants' first three years of establishment only, based on a sample drawn from a bilingual area in order to distinguish the effect of language skill from the social effect of language. How
\end{abstract}


does language affect occupational mobility (i.e. the time taken to find a job, and the duration of that job)? The effect language has on mobility varies according to the ranking of the job: language proficiency is found to shorten the duration of the first job and to extend the time spent in the third. The type of effect also changes over time: although language skill alone seems to play a part at first, this effect later tapers off and is replaced by the social effect of language. 